Rhetorische Strategien des Hofmannes 


\author{
Manfred Hinz
}

\title{
Rhetorische Strategien des Hofmannes
}

Studien zu den italienischen Hofmannstraktaten des 16. und 17. Jahrhunderts 
Die Deutsche Bibliothek - CIP-Einheitsaufnahme

Hinz, Manfred:

Rhetorische Strategien des Hofmannes :

Studien zu den italienischen Hofmannstraktaten des 16. und 17. Jahrhunderts /

Manfred Hinz. - Stuttgart : Metzler, 1992

(Romanistische Abhandlungen : 6)

ISBN 978-3-476-00820-6

ISBN 978-3-476-03395-6 (eBook)

DOI 10.1007/978-3-476-03395-6

NE: GT

Dieses Werk einschließlich aller seiner Teile ist urheberrechtlich geschützt. Jede Verwertung außerhalb der engen Grenzen des Urheberrechtsgesetzes ist ohne Zustimmung des Verlages unzulässig und strafbar. Das gilt insbesondere für Vervielfältigungen, Übersetzungen, Mikroverfilmungen und die Einspeicherung und Verarbeitung in elektronischen Systemen.

(C) 1992 Springer-Verlag GmbH Deutschland

Ursprünglich erschienen bei J. B. Metzlersche Verlagsbuchhandlung und Carl Ernst Poeschel Verlag GmbH in Stuttgart 1992 


\section{INHALT}

\section{DER HOFMANN, CHARAKTERISTIKA EINER PROFESSION}

I.1 Versuche einer Berufsbestimmung

Castiglione: die Notwendigkeit einer neuen »arte e disciplina - Garzoni: Hofmann und Schmeichler - Sabba da Castiglione: Schwierigkeiten des christlichen Hofmannes.

\section{I.2 Vom Humanisten zum Hofmann}

Zur Krisengeschichte des Kleinadels - Hofleutebiographien: Canossa, Fregoso, Castiglione - »civic humanism « vs. »umanesimo cortigiano - Dialogtheorie in Brunis Dialogus.

\section{CASTIGLIONES LIBRO DEL CORTEGIANO UND DIE BEGRÜNDUNG DER HOFMANNSKUNST}

\section{II.1 Das Spiel des Hofmannes}

Eine Enzyklopädie für Hofleute - vier Interpretationen - Traktat oder Biographie? - Speronis Dialogtheorie - das Hofmannsspiel - die perfekte Hofgesellschaft - die Prüfungen des Ingeniums - Konversationstopik - »rhetorica utens« und »rhetorica docens - die Zurückweisung der Hofkritik.

\section{II.2 Die Grenzen der Konversation} 100

Die Selbsterziehung der Hofgesellschaft - Castigliones Konversation und Dialogtheorien - Ausschluß von Bildungsinhalten - das Spontaneitätsgebot - die Relevanz der Konversationsbeiträge.

\section{II.3 Die Ausstattung des Hofmannes: »grazia« und »sprezzatura« ..... 110} Der Status der "grazia - Ausschluß von Natur (»nobilitas«) - Definitionsversuche der »grazia « - »decorum « - eine »regula universalissima « - »celare artem« - das Arbeitsverbot - Albertis Arbeitsethik - Selbstvergrößerung - "sprezzatura affettata« - »sprezzatura« als Hermeneutik. 
II.4 Hofmann und Prinz

Die Abhängigkeit des Prinzen vom Hofmann - »il fin della cortegiania « - Hofmann und Philosoph - Hofmann und Schmeichler - die Vergesellschaftung des Prinzen - die Umkehrung der Hierarchie - Funktion der Religion.

\section{II.5 Die Sprache des Hofmannes}

Linguistische Affektiertheit - Speronis Dialogo delle lingue - »acutezza recondita«

- das Problem der »imitatio - »parole« und »sentenzie« - zum Ursprung der »acutezza - »la consuetudine sia la maestra - die stilbildende Klasse.

\section{II.6 Die Poetik des Hofmannes}

Eine Poetik der kleinen Formen - die Grenzen des Komischen im De oratore Pontanos »vir facetus « - zwei Witztypen - »pronte acutezze« - die Topik des Komischen.

\section{II.7 Die Manieren des Hofmannes}

Gesellschaftliche Urteilskraft und private Vernunft - Stilhistorismus - »ingenium« und »iudicium « - »instinto naturale « - »natürliche « Urteilskraft - die »Umstände« und ihre »Örter« - die Entfaltung der Konversationstopik - das Scheitern der Konversation - eine Gesellschaft ohne Ursprungsmythos.

\section{DIE HANDBÜCHER DER HOFMANNSKUNST}

III.1 Die Verabschiedung des Prinzenerziehers

Nifos Handbuch für Schmeichler und »buffoni« - Hofmann und Prinzenunterhalter

- Grimaldi Robios Korrektur des Cortegiano - und sein Plagiat - »amoreuolezza«

- Sprechen am hierarchischen Hof - linguistische Fertigteile - die »nikodemische»

Lösung Rosellos.

III.2 Die Manualisierung des Cortegiano

Das Inhaltsverzeichnis von 1541 - Dolces »Tavola« - Indices und Regelsammlungen.

\section{III.3 Hofmann und Literat bei Giovanni Andrea Gilio da Fabriano}

Die Konkurrenz der Hofmannskandidaten bei Gilio da Fabriano - die Ausbildung des »letterato cortigiano « - die Konversationssprache - die Literatursprache - der Hofpoet bei Giraldi Cinzio.

III.4 Die Diffusion der Hofmannskunst .261

Der Hofmann als Sekretär - der Hofmann als Ritter - eine »Summa« der Hofmannskunst - die Verschlüsselung der Hofsprache - die Ununterscheidbarkeit des guten und schlechten Hofmannes - die 72 Berufe des Hofmannes. 


\section{DELLA CASAS NORMALISIERUNG GESELLSCHAFTLICHEN VERHALTENS}

\section{IV.1 De officiis und die Neutralisierung sozialer Hierarchie}

Der Galateo, ein »demokratischer « Cortegiano? - De officiis und die Nikomachische

Ethik - die sozialen Voraussetzungen der Konversation - die Konventionalisierung von Herrschaft - die Rhetorik der »inferiores amici« - Dienst als

Zivilisationsleistung - »mediocritas« und "philía«.

\section{IV.2 Soziale »belle maniere« und private Tugend}

Zur Publikationsgeschichte des Galateo - Galateo und Cortegiano - die Indifferenz von Manieren und Tugenden - »usus communis« und »habitus « - »ragione « und »costume«

- Distinktion durch Anpassung: die »misura« - das Zeremoniell - zwei

Schönheitsbegriffe.

IV.3 Die Konversationssprache des Galateo 308

Die Begrenzung der Konversationsinhalte - »motti« und Novellen - eine unsoziale Poetik: Dante - das Problem der Dialekte - die Wahl des Stils und das Vorbild Boccaccios - die Novellistik des Galateo - zur Rezeptionsgeschichte.

\section{DIE KONVERSATION ALS GESELLSCHAFTSMODELL IN LA CIVIL CONVERSATIONE VON STEFANO GUAZZO}

\section{V.1 Konversation und Konversationstheorie}

$\mathrm{Zu}$ Guazzos Biographie - Civil conversatione und Cortegiano - die Melancholie als Ausgangspunkt - der Zirkel der Konversation - die Grenzen der Konversation - Distinktion und »sprezamento«.

\section{V.2 Die soziale Ordnung der Konversation}

Die Laster und der gute Ruf - Taxonomie der Ständesprachen - der Hofmann im Hausstand des Prinzen.

\section{V.3 Konversation und Poetik}

Der metapoetische Status der Konversation - »usus communis« und Distinktion - die »motti« - »sapere co i manco, \& parlar co i più «.

\section{V.4 Die akademische Konversation}

Die Ersetzung äußerer Autoritäten - rhetorische Kombinatorik - die poetischen Gattungen der Akademie - Sprichwörter - das Madrigal - literarische Florilegien - die akademische und die höfische Konversation. 


\section{DER »TACITISTISCHE« HOFMANN BEI LORENZO} DUCCI

Seianus als Paradigma - ein methodisches Lehrbuch der Hofmannskunst - die Gesellschaftlichkeit von Prinz und Hofmann - der Zweck des Hofmannes und der des Prinzen - die Doppelnatur des Prinzen - die Vortäuschung von Affekten - die Verstellung des Prinzen und die des Hofmannes - die Konversation als Austragungsort sozialer Konflikte - Tadel und Schmeichelei - die Auflösung der Hofgesellschaft - das Dilemma der Hofmannskunst als Geheimlehre.

\section{DER »WEISE« AM HOF BEI MATTEO PEREGRINI}

\section{VII.1 Die Debatte zwischen Matteo Peregrini und Giovan Battista}

Manzini

Ein Professor als Hofmannstraktatist - der Hofmann am absolutistischen Hof

- Hofmann und Minister.

VII.2 Die Auflösung der Hofgesellschaft

Die Indirektion der Hofmannskunst - die Selbstaufgabe des Hofmannes - die Konkurrenz der Selbsterniedrigung - Ununterscheidbarkeit von Wahrheit und Verstellung - der Günstling - die Identität von Macht und Recht - der Souverän als Verkörperung des Staates - »la Corte è il metro della vita Ciuile« - »celarsi a tutti« vs. »scoprir tutti

VII.3 Die Privilegien des Weisen am Hof

Perfekte Selbstkontrolle - Ataraxie und gesellschaftlicher Erfolg - die öffentliche Funktion des Weisen - das Problem der Erlernbarkeit von Weisheit - Abschied von der »historia magistra vitae« - die Grenzen der höfischen Weisheit - der Weise und der Literat.

VII.4 Die Sprache als Medium höfischer Konkurrenz 420

Die Rhetorik der Sprachkontrolle - »Adulatione è ciuiltà - das Verbot des Lachens

- Simulation und Dissimulation - die Versachlichung der Diskussion - die

Doppeldeutigkeit der Zeichen.

VII.5 Die »acutezza« in der Hofsprache

Die »acutezza« im Kontext der Hofmannstraktatistik - die logische »acutezza« - »acutezza« als scheinbare Kunst - Ingenium und Verstand - Metapher - die sozialen Grenzen der »acutezza« - »acutezza« und Affektiertheit - »acutezza« und »aptum«. 
VII.6 Matteo Peregrini und die Aporien der Hofmannstraktatistik ......444

Das Ende der Hofmannstraktatistik - die Trennung von »rhetorica utens« und »rhetorica docens « - von der Rhetorik zur Hermeneutik - die Hofmannstraktate als Indizien historischer Prozesse - der Selbstwiderspruch der Hofmannskunst - von der stratifizierten zur funktionalen Gesellschaft - die Selbstverteidigung der Aristokratie. 